PROCEEDINGS OF THE

AMERICAN MATHEMATICAL SOCIETY

Volume 139, Number 3, March 2011, Pages 1095-1098

S 0002-9939(2010)10529-4

Article electronically published on August 6, 2010

\title{
THE DISTANCE $\operatorname{dist}(\mathcal{B}, X)$ WHEN $\mathcal{B}$ IS A BOUNDARY OF $B\left(X^{* *}\right)$
}

\author{
A. S. GRANERO, J. M. HERNÁNDEZ, AND H. PFITZNER
}

(Communicated by Nigel J. Kalton)

\begin{abstract}
Let $X$ be a real Banach space and let $\mathcal{B}$ be a boundary of the unit ball $B\left(X^{* *}\right)$ of the bidual $X^{* *}$ (which means that for each $x^{*} \in X^{*}$ there is $b \in \mathcal{B}$ such that $\left.\left\langle b, x^{*}\right\rangle=\left\|x^{*}\right\|\right)$. We show that $\operatorname{dist}(\mathcal{B}, X)=\operatorname{dist}\left(B\left(X^{* *}\right), X\right)$ where $\operatorname{dist}(A, X)$ denotes the sup of all $\operatorname{dist}(a, X)$ with $a \in A$. Since $\overline{\mathrm{co}}^{w^{*}}(\mathcal{B})=$ $B\left(X^{* *}\right)$ this is in contrast with the fact that in general strict inequality can occur between $\operatorname{dist}(K, X)$ and $\operatorname{dist}\left(\overline{\mathrm{co}} w^{*}(K), X\right)$ even for a $w^{*}$-compact $K \subset$ $X^{* *}$.
\end{abstract}

If $K$ is a $w^{*}$-compact subset of a dual Banach space $X^{*}$, a subset $\mathcal{B}$ of $K$ is said to be a (James) boundary of $K$ if every $x \in X$ attains its maximum on $K$ at an element of $\mathcal{B}$, that is to say, if for every $x \in X$ there is $b \in \mathcal{B}$ such that $\langle b, x\rangle=\sup \langle K, x\rangle$. For instance, $K$ itself and the set of extreme points of $\overline{\mathrm{co}}^{w^{*}}(K)$ (which is contained in $K$ ) are boundaries of $K$, but there are boundaries that do not meet the set of extreme points (see [3] and the references therein for boundaries).

For two sets $A, C \subset X$ and $x \in X$, $\operatorname{dist}(x, C)$ denotes the usual distance from $x$ to $C$ and $\operatorname{dist}(A, C)=\sup \{\operatorname{dist}(a, C): a \in A\}$ denotes the (non-symmetric) greatest distance from $A$ to $C$. It is easy to see that $\operatorname{dist}(A, C)=\operatorname{dist}(\bar{A}, C)$ and, if $C$ is a convex subset, then $\operatorname{dist}(A, C)=\operatorname{dist}(\overline{\mathrm{co}}(A), C)$. In this short paper we prove

Theorem 1. If $X$ is a real Banach space and $\mathcal{B}$ a boundary of the dual unit ball $B\left(X^{* *}\right)$, then $\operatorname{dist}(\mathcal{B}, X)=\operatorname{dist}\left(B\left(X^{* *}\right), X\right)$ (which equals 0 if $X$ is reflexive and 1 if $X$ is not reflexive).

Before passing to the proof we shortly explain its context and fix the notation. The topic of our theorem is part of the general question to which extent and in which sense $\overline{\mathrm{co}} w^{*}(\mathcal{B})$ can be recovered by a boundary $\mathcal{B}(\mathrm{cf} . \quad 2,3,4,5,6,9$, ). When $K$ is a $w^{*}$-compact subset of a bidual Banach space $X^{* *}$ and $\mathcal{B}=K$, the distance $\operatorname{dist}(K, X)$ is, in general, different from the distance $\operatorname{dist}\left(\overline{\mathrm{co}}^{w^{*}}(K), X\right)$. Actually, it is known (see [2, 5]) that

$$
\operatorname{dist}\left(\overline{\mathrm{co}}^{w^{*}}(K), X\right) \leq 5 \operatorname{dist}(K, X),
$$

while [6, Prop. 10] exhibits a Banach space $X$ and a $w^{*}$-compact subset $K \subset X^{* *}$ such that $\operatorname{dist}\left(\overline{\mathrm{co}} w^{*}(K), X\right)=3 \operatorname{dist}(K, X)>0$, which shows that the factor 5 above

Received by the editors February 26, 2010 and, in revised form, April 6, 2010.

2010 Mathematics Subject Classification. Primary 46B20; Secondary 46B26.

Key words and phrases. Convex sets, James boundaries, unit ball, distances.

This work was supported in part by grant DGICYT MTM2005-00082, grant UCM-910346 and grant UCM-BSCH PR27/05-14045.

(C)2010 American Mathematical Society Reverts to public domain 28 years from publication 
cannot be replaced by 1 . It is not known whether (0.1) holds if $K$ is replaced by an arbitrary boundary $\mathcal{B}$ of $\overline{\mathrm{co}} w^{*}(\mathcal{B})$. In the special case $\overline{\mathrm{co}} w^{*}(K)=B\left(X^{* *}\right)$, however, our theorem shows that (0.1) holds with 1 instead of 5 and for any boundary $\mathcal{B}$ of $B\left(X^{* *}\right)$. Note that in this case one has $\operatorname{dist}\left(B\left(X^{* *}\right), X\right)=0$ if $X$ is reflexive and $\operatorname{dist}\left(B\left(X^{* *}\right), X\right)=1$ if $X$ is not reflexive.

Our notation is standard (cf. [7, 1]). The underlying scalar field we consider is the reals. If $(X,\|\cdot\|)$ is a Banach space, let $B(X)$ and $S(X)$ be the closed unit ball and unit sphere of $X$, respectively, and $X^{*}$ its topological dual. The weak*topology of the dual Banach space $X^{*}$ is denoted by $w^{*}$ and the weak topology of $X$ by $w \cdot \operatorname{co}(A)$ denotes the convex hull of the set $A, \overline{\operatorname{co}}(A)$ is the $\|\cdot\|$-closure of $\operatorname{co}(A)$ and $\overline{\mathrm{co}}^{w^{*}}(A)$ the $w^{*}$-closure of $\operatorname{co}(A)$. An $\ell_{1}$-sequence is a sequence which is equivalent to the canonical basis of $\ell_{1}=\ell_{1}(\mathbb{N})$. A Banach space $X$ is said to contain an asymptotically $y^{1}$ isometric copy of $\ell_{1}$ if there exists an $\ell_{1}$-sequence $\left(a_{n}\right)$ in $B(X)$ and a sequence $\left.\left.\left(\delta_{n}\right)_{n \in \mathbb{N}} \subset\right] 0,1\right]$ converging to 1 satisfying the following inequality for every finite sequence $\left(\lambda_{i}\right)_{1 \leq i \leq n}$ of $\mathbb{R}$ :

$$
\sum_{1 \leq i \leq n} \delta_{i}\left|\lambda_{i}\right| \leq\left\|\sum_{1 \leq i \leq n} \lambda_{i} a_{i}\right\|
$$

Given a sequence $\left(x_{n}\right)$ in a Banach space $X$ we call $\left(\sum_{i \in F_{n}} \lambda_{i} f_{i}\right)$ a block sequence of $\left(x_{n}\right)$ if $\left(F_{n}\right)$ is a sequence of finite pairwise disjoint subsets of $\mathbb{N}$ and $\left(\lambda_{i}\right)_{i \in \mathbb{N}}$ is a sequence of scalars such that $\sum_{i \in F_{n}}\left|\lambda_{i}\right|=1$ for all $n \in \mathbb{N}$. The dual unit ball of a Banach space $X$ is said to be $w^{*}$-block compact if each sequence in $B\left(X^{*}\right)$ admits a $w^{*}$-convergent block sequence.

Proof of Theorem 1. We will distinguish the two cases that $X$ contains an asymptotically isometric copy of $\ell_{1}$ and that it does not. This distinction comes naturally from Morillon's proof [8] of James' theorem.

First case: $X$ contains an asymptotically isometric copy of $\ell_{1}$. We simply adapt the proof of [8, Th. 3]:

Proposition 2. Let $X$ be a Banach space containing an asymptotically isometric copy of $\ell_{1}$ and let $\mathcal{B}$ be a boundary of $B\left(X^{* *}\right)$. Then there exists $b \in \mathcal{B}$ such that $\operatorname{dist}(b, X)=\operatorname{dist}\left(B\left(X^{* *}\right), X\right)$ and $\operatorname{so} \operatorname{dist}(\mathcal{B}, X)=\operatorname{dist}\left(B\left(X^{* *}\right), X\right)$.

Proof. Let $\left(a_{i}\right)_{i \in \mathbb{N}} \subset B(X)$ be an asymptotically isometric copy of $\ell_{1}$. As in 8 , Th. 3] let $g_{n} \in X^{*}$ be, for each $n \in \mathbb{N}$, a Hahn-Banach extension to $X$ of the functional on $\overline{\left[\left(a_{i}\right)_{i \in \mathbb{N}}\right]}$ defined by $a_{i} \mapsto-\delta_{i}$ if $i<n$, and $a_{i} \mapsto \delta_{i}$ if $i \geq n$. Then $\left\|g_{n}\right\| \leq 1$ by $(0.2)$. Let $g \in X^{*}$ be a $w^{*}$-cluster point of $\left\{g_{n}: n \geq 1\right\}$ and set $h=\left(\sum_{k \in \mathbb{N}} 2^{-k} g_{k}\right)-g$. Clearly $\|h\| \leq 2$ because $\|g\| \leq 1$ but also $\|h\| \geq 2$ because by construction

$$
\sup _{i \in \mathbb{N}}\left\langle h, a_{i}\right\rangle=\sup _{i \in \mathbb{N}} \delta_{i}\left(\sum_{n \leq i} 2^{-n}-\sum_{i<n} 2^{-n}+1\right)=2 .
$$

Since $\mathcal{B}$ is a boundary of $B\left(X^{* *}\right)$ there is $b \in \mathcal{B}$ such that $\langle b, h\rangle=2$ and so $\left\langle b, g_{n}\right\rangle=1$ for all $n \in \mathbb{N}$ and $\langle b, g\rangle=-1$. Let $x \in X$. If either $\langle g, x\rangle \geq 0$ or $\langle g, x\rangle \leq-2$, then $\|b-x\| \geq|\langle b-x, g\rangle| \geq 1$. Otherwise, $-2<\langle g, x\rangle<0$ and there exists some $m \in \mathbb{N}$ (depending on $x$ ) such that $-2<\left\langle g_{m}, x\right\rangle<0$. Hence $-3<\left\langle x-b, g_{m}\right\rangle<-1$ and

\footnotetext{
${ }^{1}$ In the literature the notion "asymptotic $l^{p}$ " can have a different meaning.
} 
again $\|b-x\| \geq\left\langle b-x, g_{m}\right\rangle>1$. This shows that $\operatorname{dist}(b, X)=1$ and completes the proof.

Second case: $X$ does not contain an asymptotically isometric copy of $\ell_{1}$.

Lemma 3. Let $X$ be a non-reflexive Banach space without an asymptotically isometric copy of $\ell_{1}$. Then

(1) $X^{*}$ contains a $w^{*}$-null sequence that does not converge weakly;

(2) for each $\epsilon>0$ there are an element $z_{\epsilon} \in S\left(X^{* *}\right)$ and a sequence $\left(f_{n}\right)$ in $B\left(X^{*}\right)$ such that $f_{n} \stackrel{w^{*}}{\rightarrow} 0$ and $\left\langle z_{\epsilon}, f_{n}\right\rangle \geq 1-\epsilon$ for all $n \in \mathbb{N}$.

Proof. (11) Except for some routine arguments, we use essentially [8, Th. 2] for this first part of the lemma. Suppose that $X$ has no asymptotically isometric copy of $\ell_{1}$ and that in $X^{*}$ each $w^{*}$-null sequence converges weakly. It follows from [8, Th. 2 ] that a Banach space without an asymptotically isometric copy of $\ell_{1}$ has a $w^{*}$ block compact dual unit ball. Therefore, since a block sequence of an $\ell_{1}$-sequence is again an $\ell_{1}$-sequence and since an $\ell_{1}$-sequence cannot converge weakly, the dual $X^{*}$ does not contain copies of $\ell_{1}$; moreover, via Eberlein-Šmulian's theorem, the dual unit ball is weakly compact because by Rosenthal's $\ell_{1}$-Theorem each sequence in it admits a weak Cauchy subsequence which $w^{*}$-converges and hence converges weakly. Hence $X$ is reflexive, which proves (11).

(2) Fix $\epsilon>0$. By (1) of this lemma there exists a $w^{*}$-null sequence $\left(x_{n}^{*}\right)$ in $B\left(X^{*}\right)$ that does not converge weakly. So, passing to a subsequence if necessary, there exist $z \in S\left(X^{* *}\right)$ and $\epsilon_{0}>0$ such that $\left\langle z, x_{n}^{*}\right\rangle \geq \epsilon_{0}>0, \forall n \geq 1$. For each $n \geq 1$ we define $a(n)$ as follows:

$$
a(n):=\inf \left\{\left\|x^{*}\right\|: x^{*} \in \operatorname{co}\left(\left\{x_{k}^{*}: k \geq n\right\}\right)\right\} .
$$

Obviously, $\epsilon_{0} \leq a(n) \leq a(n+1) \leq 1$. Let

$$
a:=\lim _{n \geq 1} a(n)=\sup _{n \geq 1} a(n) .
$$

We choose $\eta>0$ and $n_{0} \in \mathbb{N}$ such that $\frac{a-\eta}{a+\eta}>1-\epsilon$ and $a-\eta<a\left(n_{0}\right)$. Now for each $k \in \mathbb{N}$ pick $v_{k} \in \operatorname{co}\left(\left\{x_{n}^{*}: n \geq n_{0}+k\right\}\right)$ so that $a\left(n_{0}+k\right) \leq\left\|v_{k}\right\| \leq a\left(n_{0}+k\right)+\eta$. Thus for each $k \geq 1$ we have

$$
a-\eta<a\left(n_{0}\right) \leq a\left(n_{0}+k\right) \leq\left\|v_{k}\right\| \leq a\left(n_{0}+k\right)+\eta \leq a+\eta .
$$

Let $f_{k}:=v_{k} /(a+\eta), \forall k \in \mathbb{N}$. Observe that $\left\|f_{k}\right\| \leq 1$. Then:

(a) By the definition of $a\left(n_{0}\right)$ and $\left(f_{k}\right)$, every $u \in \operatorname{co}\left(\left\{f_{k}: k \geq 1\right\}\right)$ satisfies $u \in \operatorname{co}\left(\left\{x_{n}^{*} /(a+\eta): n \geq n_{0}\right\}\right)$ and so

$$
1 \geq\|u\| \geq \frac{a\left(n_{0}\right)}{a+\eta}>\frac{a-\eta}{a+\eta} \geq 1-\epsilon .
$$

Thus, $\operatorname{co}\left(\left\{f_{k}: k \geq 1\right\}\right) \cap \frac{a-\eta}{a+\eta} B\left(X^{*}\right)=\emptyset$. By the Hahn-Banach separation theorem there exists $z_{\epsilon} \in S\left(X^{* *}\right)$ such that $\left\langle z_{\epsilon}, u\right\rangle \geq(a-\eta) /(a+\eta) \geq 1-\epsilon$ for every $u \in \operatorname{co}\left(\left\{f_{k}: k \geq 1\right\}\right)$.

(b) Since $f_{k}$ is a finite convex combination of elements of $\left\{x_{n}^{*} /(a+\eta): n \geq n_{0}+k\right\}$ and $x_{n}^{*} \stackrel{w^{*}}{\rightarrow} 0$, necessarily $f_{k} \stackrel{w^{*}}{\rightarrow} 0$.

Remark. The second part of Lemma 3 gives an improvement (probably known as folklore) of the Josefson-Nissenzweig Theorem for non-Grothendieck spaces. (A 
Grothendieck space is a Banach space in whose dual $w^{*}$-convergent sequences converge weakly.)

Proposition 4. Let $X$ be a Banach space without an asymptotically isometric copy of $\ell_{1}$ and let $\mathcal{B}$ be a boundary of $B\left(X^{* *}\right)$. Then $\operatorname{dist}(\mathcal{B}, X)=\operatorname{dist}\left(B\left(X^{* *}\right), X\right)$.

Proof. If $X$ is reflexive, the statement is trivially true. Assume that $X$ is not reflexive. Let $\epsilon>0$ and choose a $w^{*}$-null sequence $\left(f_{n}\right)_{n \geq 1} \subset B\left(X^{*}\right)$ according to Lemma 3, By Simons' equality [9, p. 69] we have

$$
\sup \left\{\limsup _{n \rightarrow \infty}\left\langle b, f_{n}\right\rangle: b \in \mathcal{B}\right\}=\sup \left\{\limsup _{n \rightarrow \infty}\left\langle z, f_{n}\right\rangle: z \in B\left(X^{* *}\right)\right\} .
$$

This expression is at least $1-\epsilon$ by Lemma 3. Hence there is $b \in \mathcal{B}$ such that $\limsup _{n \rightarrow \infty}\left\langle b, f_{n}\right\rangle>1-2 \epsilon$ and so there is a subsequence $\left(f_{n_{k}}\right)_{k \geq 1}$ such that $\left\langle b, f_{n_{k}}\right\rangle>1-2 \epsilon$ for all $k$. Let $v$ be a $w^{*}$-cluster point of $\left\{f_{n_{k}}: k \geq 1\right\}$ in $X^{* * *}$. Then $v \in X^{\perp}$ because $\left(f_{n_{k}}\right)_{k \geq 1}$ is $w^{*}$-null in $X^{*}$. Furthermore $\|v\| \leq 1$ and $\langle v, b\rangle \geq 1-2 \epsilon$. Now for any $x \in X$ we have that $\|b-x\| \geq\langle v, b-x\rangle=\langle v, b\rangle \geq 1-2 \epsilon$; hence $\operatorname{dist}(b, X) \geq 1-2 \epsilon$. Since $\epsilon$ was arbitrary this proves the proposition.

Theorem 1 is clear from the two propositions.

\section{REFERENCES}

[1] J. Diestel, Sequences and Series in Banach Spaces, Springer-Verlag, Berlin-HeidelbergNew York, 1984. MR737004 (85i:46020)

[2] M. Fabian, P. HájeK, V. Montesinos And W. Zizler, A quantitative version of Krein's theorem, Rev. Mat. Iberoam., 21(1) (2005), 237-248. MR2155020 (2006b:46011)

[3] V.P. Fonf, J. Lindenstrauss, and R.R. Phelps, Infinite dimensional convexity, Chapter 15 of W. B. Johnson and J. Lindenstrauss, Handbook of the Geometry of Banach Spaces, Volumes 1 and 2, North Holland, 2001, 2003. MR.1863703 (2003c:46014)

[4] G. Godefroy, Boundaries of a convex set and interpolation sets, Math. Ann., 277(1987), 173-184. MR886417 (88f:46037)

[5] A. S. Granero, An extension of the Krein-Šmulian theorem, Rev. Mat. Iberoam., 22 (1) (2006), 93-110. MR2267314 (2008a:46019)

[6] A. S. Granero, P. Hásek and V. Montesinos, Convexity and $w^{*}$-compactness in Banach spaces, Math. Ann., 328 (2004), 625-631. MR2047643 (2005c:46020)

[7] W. B. Johnson and J. Lindenstrauss, Handbook of the Geometry of Banach Spaces, Volumes 1 and 2, North-Holland, 2001, 2003. MR1863689 (2003f:46013)

[8] M. Morillon, A new proof of James' sup theorem, Extracta Math., 20 (2005), 261-271. MR2243342 (2007h:46014)

[9] S. Simons, An eigenvector proof of Fatou's lemma for continuous functions, Math. Intel., 17(1995), 67-70. MR1347898 (96e:26003)

Departamento de Análisis Matemático, Facultad de Matemáticas, Universidad ComPlutense De MADRID, 28040-Madrid, Spain

E-mail address: AS_granero@mat.ucm.es

Departamento de Análisis Matemático, Facultad de Matemáticas, Universidad ComPLUTENSE DE MADRID, 28040-MADRID, SPAin

E-mail address: juanmanuel_hrl@hotmail.com

Université D'Orléans, BP 6759, F-45067, Orléans Cedex 2, France

E-mail address: Hermann.Pfitzner@univ-orleans.fr 